\title{
Variable patterns of ST-T abnormalities in patients with left ventricular hypertrophy and normal coronary arteries
}

\author{
F U Huwez, S D Pringle, P W Macfarlane
}

\begin{abstract}
Background-Classically, the ST-T configuration in the electrocardiogram of patients with left ventricular hypertrophy is said to have a typical pattern of ST depression together with asymmetrical $T$ wave inversion (the so-called left ventricular strain pattern). However, many patients with left ventricular hypertrophy may also have ischaemic heart disease. To revise the electrocardiographic criteria for left ventricular hypertrophy the ST-T configuration in patients with left ventricular hypertrophy documented by echocardiography and with normal coronary arteries was assessed.
\end{abstract}

Methods-24 patients were selected for this study. All had left ventricular hypertrophy documented by echocardiography, normal coronary arteries by cardiac catheterisation, and ST and/or $T$ wave abnormalities in the lateral leads of their electrocardiogram. There were eight patients with aortic valve disease and 16 with hypertension who had coronary angiography as part of an investigation into the risk factors of sudden cardiac death caused by hypertensive left ventricular hypertrophy. No patient was receiving digitalis preparations or had electrolyte disturbances, and none had a previous myocardial infarction or ventricular conduction defect.

Results-Typical electrocardiographic evidence of left ventricular strain was found in approximately two thirds (63\%) of patients and $95 \%$ of this subgroup had asymmetrical $T$ wave inversion. Flat $S T$ segment depression, with or without $T$ wave inversion or isolated $T$ wave inversion (symmetrical or asymmetrical) in the anterolateral leads, was seen in the remaining $37 \%$ of patients.

Conclusions-These findings indicate that left ventricular hypertrophy without coronary artery disease can cause variable types of ST-T abnormalities in the anterolateral leads including the typical left ventricular strain pattern and non-specific ST-T changes. Nonspecific abnormalities could not be distinguished from those of coronary artery disease and may adversely affect the accuracy of the electrocardiographic criteria for the diagnosis of left ventricular hypertrophy because they do not accord with the criteria for left ventricular strain.

Carter and Estes showed that electrocardiographic evidence of left ventricular strain defined as asymmetrical ST depression and T wave inversion in the anterolateral leads ( $\mathrm{I}$, aVL, V5 and/or V6) correlated significantly with left ventricular weight at necropsy. ${ }^{1}$ Left ventricular strain (ST segment depression $\leq 0.1 \mathrm{mV}$ with asymmetrical $\mathrm{T}$ wave inversion) in the anterolateral leads was reported to be $95 \%$ specific for left ventricular hypertrophy in the absence of digitalis treatment. ${ }^{2}$ However, in clinical practice, it is not uncommon to find that some patients, who have electrocardiographic left ventricular hypertrophy confirmed by echocardiography, have symmetrical or asymmetrical $T$ wave inversion without ST segment depression. It is not certain whether such $T$ wave changes without ST-segment depression occur because of left ventricular hypertrophy itself or on account of associated coronary artery disease particularly as it is well established that patients with electrocardiographic left ventricular hypertrophy and ST-T abnormalities are eight times more likely to have coronary artery disease than those who have electrocardiographic left ventricular hypertrophy without repolarisation wave abnormalities. ${ }^{3}$ For this reason, we decided to review the patterns of repolarisation wave abnormalities in a group of patients with left ventricular hypertrophy documented by echocardiography, ST and/or $T$ wave abnormalities on their scalar electrocardiograms and with normal coronary arteries demonstrated by coronary angiography.

Patients and methods

Patients were selected for this study if they had normal coronary arteries and left ventricular hypertrophy documented by echocardiography. Their electrocardiograms also had to show ST and/or T wave abnormalities in the lateral leads. Eight patients had aortic valve disease (aortic stenosis and/or regurgitation) with or without chest pain suggestive of angina pectoris and had left heart catheterisation and coronary angiography as a part of their general assessment for aortic valve replacement. Sixteen patients had hypertension and they had coronary angiography as a part of an ongoing study investigating the risk 
factors of sudden cardiac death in hypertensive left ventricular hypertrophy. ${ }^{4}$ No patient had a previous myocardial infarction, intraventricular conduction defect, or was being treated with digitalis. Myocardial infarction was excluded in these patients on the basis of clinical history and contrast left ventriculography.

The electrocardiograms were recorded with either a Mingorec 4 electrocardiograph (Siemens-Elema) or a computer compatible electrocardiograph developed locally. ${ }^{5}$ ST segment depression was regarded as present when depression $\leq 0.1 \mathrm{mV}$ occurred $40 \mathrm{~ms}$ after the J point. The configuration of the ST$T$ changes was classified according to the following rules:

ST segment depression-downward sloping, flat or upward sloping.

$T$ wave inversion-symmetrical, or asymmetrical with or without terminal overshoot.

The echocardiograms were recorded with a Diasonics Cardiovue 3400 Phased Array imaging system and a $2.5 \mathrm{MHz}$ transducer. Patients were positioned semisupine in the $45^{\circ}$ left lateral position and the echo windows that best demonstrated the septal and posterior walls of the left ventricle were chosen in the 3rd, 4th, or 5th left parasternal area. $M$ mode recordings were obtained and measurements were made of the interventricular septum, posterior wall of the left ventricle, and the left ventricular internal dimension at the tip of the mitral valve at end diastole according to the Penn Convention. ${ }^{6}$ The left ventricular masses were calculated by the formula of Devereux and Reichek ${ }^{6}$ as follows:

$$
\begin{aligned}
& \text { LVM }= \\
& 1.04[(\text { IVS } \\
& \text { (LVIDD) }]-13.6 \mathrm{~g}
\end{aligned}
$$

where LVM is the left ventricular mass ( $g$ ), IVS is the interventricular septum $(\mathrm{cm})$, PWLV is the posterior wall of left ventricle (cm), and LVIDD is the left ventricular internal dimension ( $\mathrm{cm}$ ) at end diastole. The masses were indexed to the body surface area, the latter being obtained from the following equation ${ }^{7}$ :

$$
\text { BSA }=0.0001 \times 71.84 \times \mathrm{Wt}^{0.425} \times \mathrm{Ht}^{0.725}
$$

where BSA is the body surface area $\left(\mathrm{m}^{2}\right)$, weight $(\mathrm{Wt})(\mathrm{kg})$, and height $(\mathrm{Ht})(\mathrm{cm})$.

Echocardiographic left ventricular hypertrophy was diagnosed when the left ventricular mass index exceeded $131 \mathrm{~g} / \mathrm{m}^{2}$ and $108 \mathrm{~g} / \mathrm{m}^{2}$ in men and women respectively. ${ }^{8}$ Absence of coronary artery disease was defined as absolutely normal coronary arteries.

\section{Results}

Twenty four patients completed the study (18 men and six women, mean (SD) age 53.2 (10.46) years, range 31-72 years). All had echocardiographic left ventricular hypertrophy and their left ventricular mass indexed to body surface area ranged from 137 to $307 \mathrm{~g} / \mathrm{m}^{2}$ with a mean (SD) of $189.34(53.7) \mathrm{g} / \mathrm{m}^{2}$. Left ventricular hypertrophy was caused by hypertension in $16 / 24(66 \%)$ and aortic valve disease (aortic stenosis and/or regurgitation) in $8 / 24$ $(34 \%)$ of the patients. All had absolutely normal coronary arteries. The table shows their left ventricular mass indexed to body surface area $\left(\mathrm{g} / \mathrm{m}^{2}\right), \mathrm{QRS}$ axes, and pattern of ST segment depression and $T$ wave inversion.

Asymmetrical ST-segment depression that was bowed upward in the anterolateral leads was seen in $15 / 24(63 \%)$ of the patients. All had $T$ wave inversions in the anterolateral leads except one patient who had diphasic $T$ waves in leads $I$, V5, and V6 (fig 1 ). The $T$ wave inversion $(\leq 0.1 \mathrm{mV})$ in the anterolateral leads of the other $14 / 24(58 \%)$ patients was predominantly asymmetrical as shown in fig 2 .

Flat ST segment depression in the anterolateral lead was found in 5/24 $(21 \%)$ of the patients (fig 3 ). The associated $T$ wave changes in the patients included asymmetrical

\begin{tabular}{|c|c|c|c|c|c|c|c|c|}
\hline No & Sex & Age (y) & $\operatorname{LVMI}\left(\mathrm{g} / \mathrm{m}^{2}\right)$ & Diag & $E C G L V H$ & $S T$ segment depression & $T$ wave inversion & $Q R S$ axis \\
\hline $\begin{array}{r}1 \\
2 \\
3 \\
4 \\
5 \\
6 \\
7 \\
8 \\
9 \\
10 \\
11 \\
12 \\
13 \\
14 \\
15 \\
16 \\
17 \\
18 \\
19 \\
20 \\
21 \\
22 \\
23 \\
24\end{array}$ & $\begin{array}{l}\mathbf{M} \\
\mathbf{F} \\
\mathbf{M} \\
\mathbf{F} \\
\mathbf{M} \\
\mathbf{M} \\
\mathbf{M} \\
\mathbf{M} \\
\mathbf{M} \\
\mathbf{M} \\
\mathbf{M} \\
\mathbf{F} \\
\mathbf{F} \\
\mathbf{M} \\
\mathbf{M} \\
\mathbf{F} \\
\mathbf{M} \\
\mathbf{M} \\
\mathbf{M} \\
\mathbf{M} \\
\mathbf{M} \\
\mathbf{M} \\
\mathbf{F} \\
\mathbf{M}\end{array}$ & $\begin{array}{l}58 \\
52 \\
45 \\
38 \\
53 \\
31 \\
63 \\
62 \\
39 \\
55 \\
53 \\
46 \\
53 \\
54 \\
52 \\
54 \\
56 \\
36 \\
72 \\
56 \\
69 \\
75 \\
52 \\
63\end{array}$ & $\begin{array}{l}169 \cdot 6 \\
127 \cdot 8 \\
162 \cdot 3 \\
193 \\
145 \\
137 \\
180 \cdot 1 \\
216 \cdot 0 \\
144 \\
245 \\
145 \\
126 \cdot 1 \\
140 \\
193 \cdot 3 \\
193 \\
305 \\
220 \\
155 \\
197 \\
246 \\
307 \\
137 \\
274 \\
186\end{array}$ & 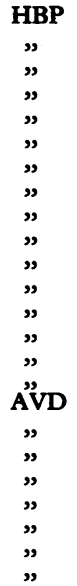 & $\begin{array}{l}- \\
+ \\
- \\
- \\
+ \\
+ \\
+ \\
- \\
+ \\
+ \\
+ \\
+ \\
+ \\
+ \\
+ \\
+ \\
+ \\
+ \\
+ \\
+ \\
+\end{array}$ & 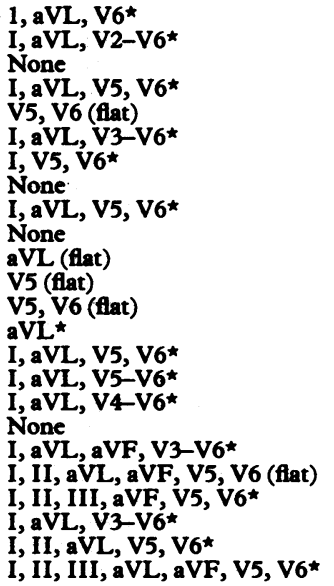 & 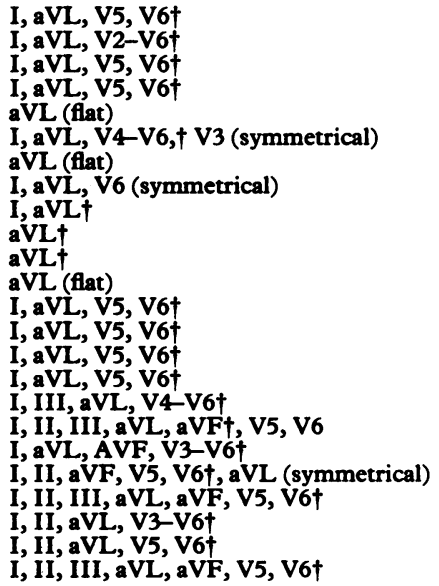 & $\begin{array}{r}30 \\
12 \\
16 \\
43 \\
35 \\
28 \\
66 \\
36 \\
03 \\
-05 \\
17 \\
67 \\
-07 \\
25 \\
14 \\
01 \\
-01 \\
42 \\
04 \\
15 \\
46 \\
64 \\
18 \\
-16\end{array}$ \\
\hline
\end{tabular}
$T$ wave inversion in two patients, flat $T$ wave in

Electrocardiographic findings in 24 patients with an increase in indexed left ventricular mass

$\star$ Downward sloping ST depression ( $\leq 0.1 \mathrm{mV}$ ). $40 \mathrm{~ms}$ after the J point.

Asymmetrical $T$ wave inversion with terminal overshoot.

HBP, high blood pressure; AVD, aortic valve disease; LVMI, left ventricular mass index; LVH, left ventricular hypertrophy. 


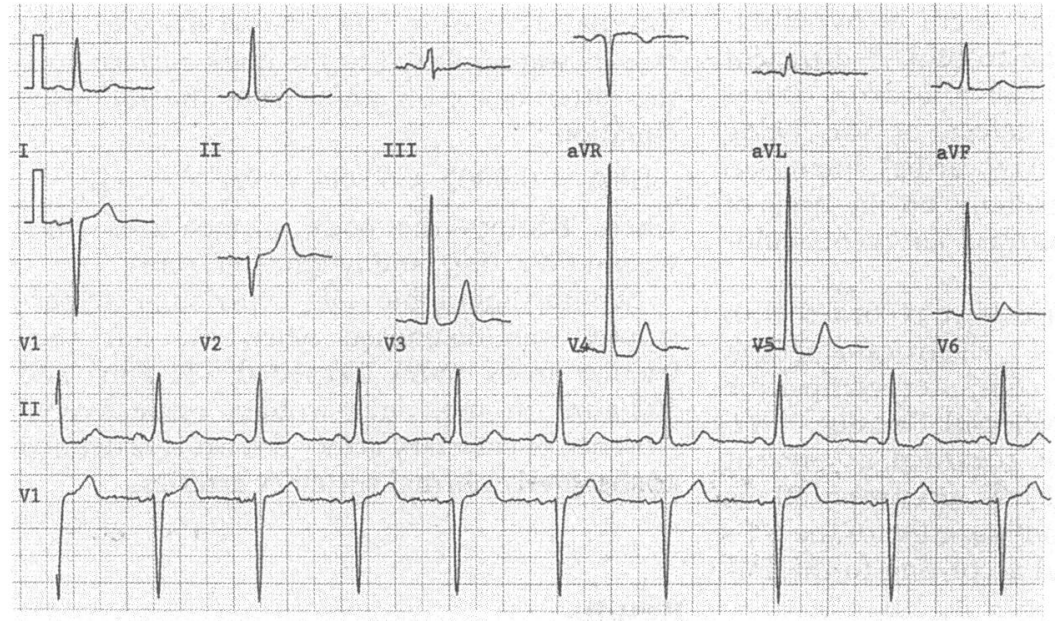

Figure $1 S T$ depression and diphasic $T$ waves in $I, V 5, V 6$ in a 53 year old man with hypertension and normal coronary arteries. His echocardiographic left ventricular mass was $145 \mathrm{~g} / \mathrm{m}^{2}$.

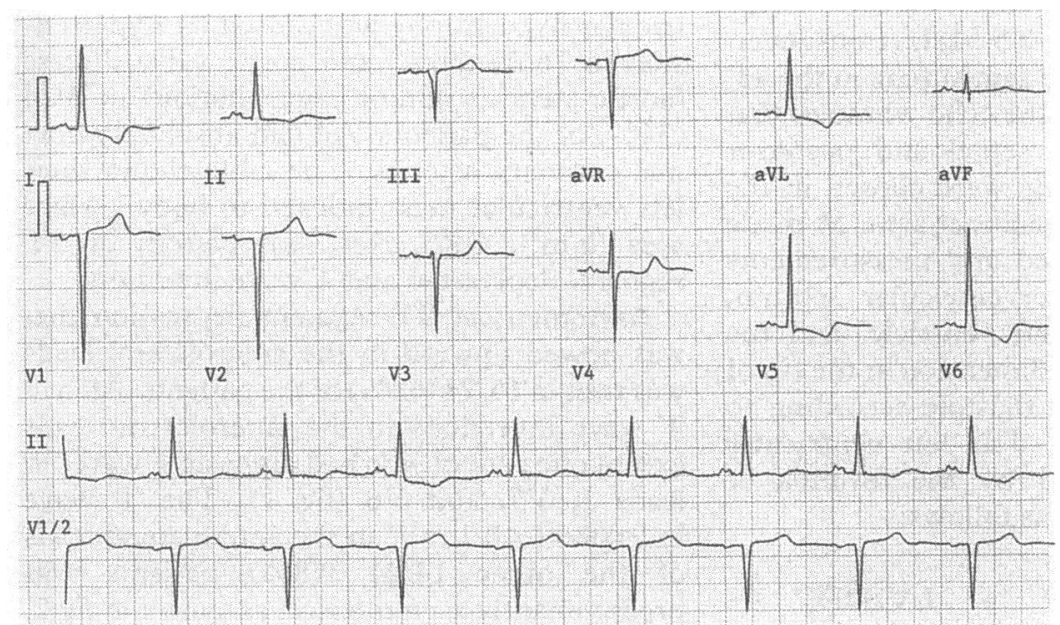

Figure 2 Electrocardiogram of a 52 year old woman with aortic valve disease and normal coronary arteries. Her echocardiographic left ventricular mass was $274 \mathrm{~g} / \mathrm{m}^{2}$. The electrocardiogram shows typical left ventricular strain with bowing upwards and sloping down into an inverted $T$ wave with terminal overshoot in the anterolateral leads.

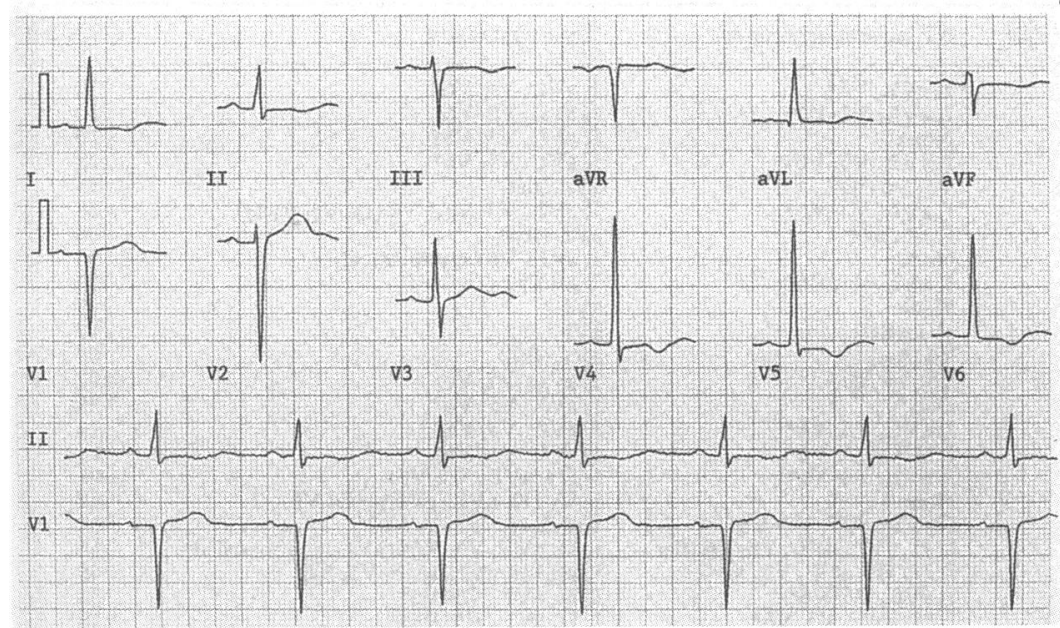

Figure 3 Flat ST segment depression in left ventricular hypertrophy without coronary artery disease in a 53 year old woman with hypertension. Her echocardiographic left ventricular mass was $140 \mathrm{~g} / \mathrm{m}^{2}$. The electrocardiogram shows $T$ wave inversion in the anterolateral leads with flat ST segment depression. two other patients, and symmetrical $T$ wave inversion in one patient.

There were isolated $T$ wave changes $(\leq 0.00$ $\mathrm{mV}$ ) without $\mathrm{ST}$ depression in the anterolateral leads in $4 / 24(16 \%)$ of the patients: three $(12 \%)$ patients with asymmetrical $T$ wave inversion (fig 4) and one (4\%) with mainly symmetrical $\mathrm{T}$ wave inversion in leads V5 and V6 (figure 5).

\section{Discussion}

The generally accepted definition of left ventricular strain is an ST segment depression that is bowed upward and slopes down into an inverted asymmetrical $T$ wave, ${ }^{9}$ a concept that was introduced by Rykert and Hepburn. ${ }^{10}$ Beach et al suggested that the repolarisation wave abnormalities of left ventricular hypertrophy without coronary artery disease could often be distinguished from those of coronary artery disease by the presence of one or more of the following five features: depression of the $J$ point, asymmetry of the $T$ wave with rapid return to the baseline, terminal positivity of the $T$ wave (overshoot), $T$ wave inversion in V6 greater than $0.3 \mathrm{mV}$, and $\mathrm{T}$ wave inversion greater in V6 than in V4. ${ }^{11}$ However, it is not rare in clinical practice to see ST-T abnormalities associated with left ventricular hypertrophy that do not fulfil these criteria of left ventricular strain and thus may be confused with those of coronary artery disease. The latter issue is further complicated by the fact that patients with left ventricular hypertrophy and ST-T abnormalities are more at risk of coronary artery disease than those who do not have abnormal repolarisation. ${ }^{3}$

In this study $15 / 24(63 \%)$ of the patients had asymmetrical ST segment depression (convex upward and sloping downwards) and 14/15 $(95 \%)$ of these patients had asymmetrical $\mathrm{T}$ wave inversion. However, flat ST segment depression with asymmetrical $\mathrm{T}$ wave inversion was observed in other $(5 / 24,21 \%)$ patients with left ventricular hypertrophy and normal coronary arteries. Furthermore, isolated $T$ wave changes (asymmetrical or symmetrical inversion) were seen in four of the 24 -that is, $16 \%$ of the repolarisation wave abnormalities associated with left ventricular hypertrophy and normal coronary arteries.

The mechanism for these ST-T abnormalities that do not accord with the definition of left ventricular strain is difficult to explain but it is well established that myocardial ischaemia may present on the surface electrocardiogram as flat ST segment depression, flat $T$ waves, symmetrical $T$ inversion, or non-specific ST-T abnormalities. ${ }^{12}$ None of these patients was taking digitalis or had electrolyte disturbances to account for the ST-T changes. Furthermore, the absolutely normal coronary arteries, documented by coronary angiography, exclude coronary artery disease as a possible explanation. However, left ventricular hypertrophy with normal coronary arteries is a known cause of angina pectoris presumed to be due to an abnormal coronary reserve which leads to relative coronary insufficiency. ${ }^{13}$ Therefore, the most likely explanation for flat ST segment 


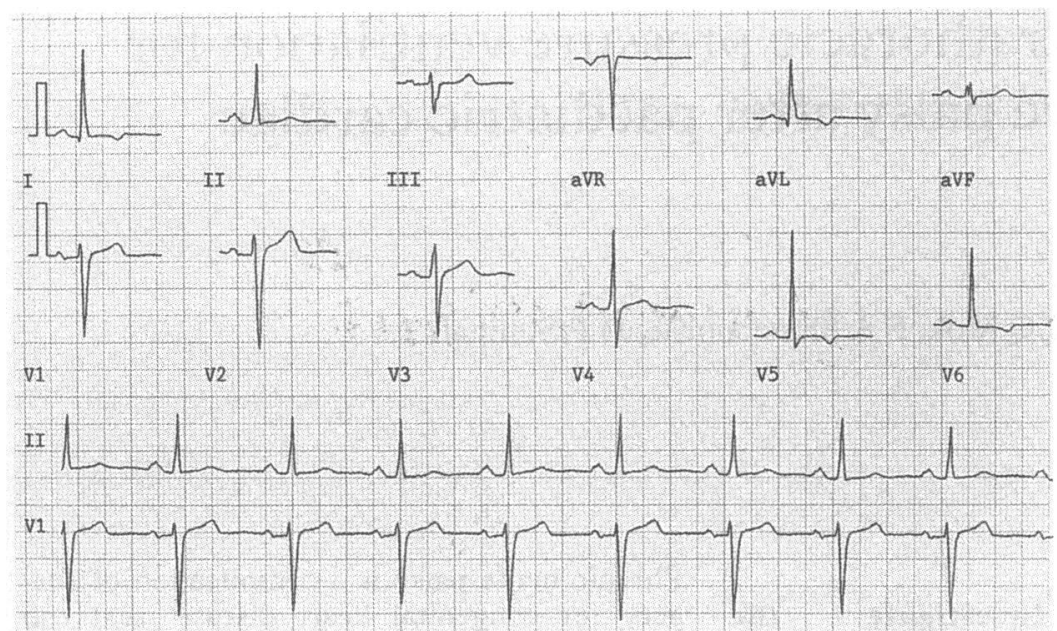

Figure 4 Electrocardiogram of a 45 year old man with left ventricular hypertrophy caused by hypertension (echocardiographic left ventricular mass of $162 \cdot 3 \mathrm{~g} / \mathrm{m}^{2}$ ). It shows mainly asymmetrical $T$ wave inversion in the anterolateral leads without $S T$ depression.

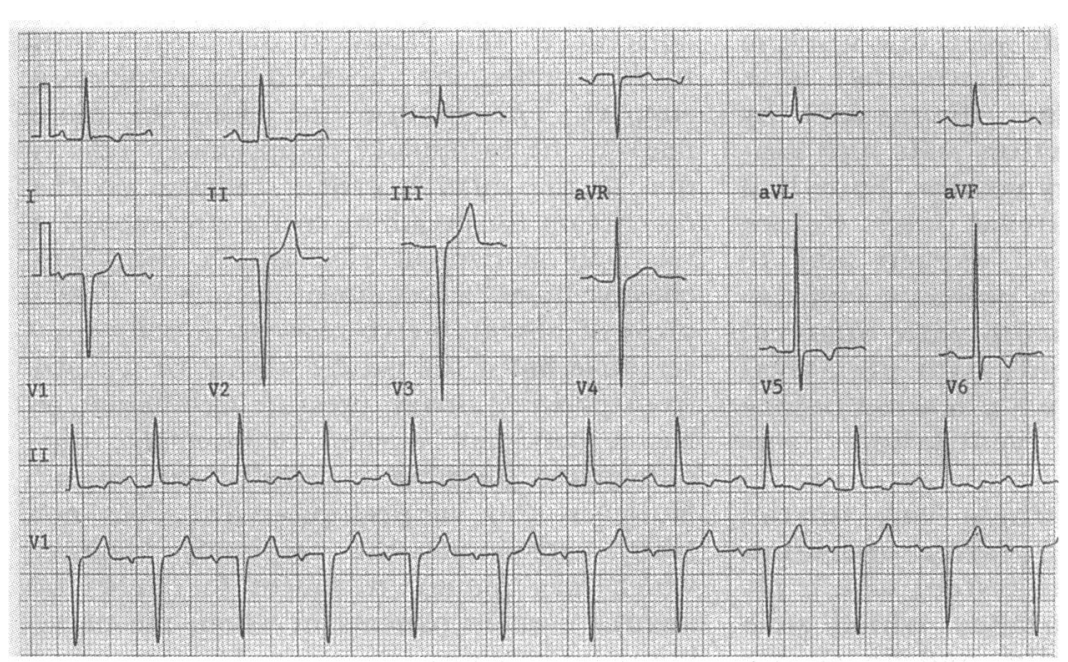

Figure 5 Electrocardiogram of a 36 year old man with mixed aortic valve disease and normal coronary arteries. It shows $T$ wave inversion without significant $S T$ segment depression in the anterolateral leads. His echocardiographic left ventricular mass was $155 \mathrm{~g} / \mathrm{m}^{2}$. such as interstitial fibrosis, as a cause of primary non-specific ST-T abnormalities in these patients with left ventricular hypertrophy and normal coronary arteries.

We conclude that typical left ventricular strain defined as asymmetrical ST segment depression ( $\leq 0.1 \mathrm{mV}, 40 \mathrm{~ms}$ after the J point) with $T$ wave inversion occurs in approximately two thirds of patients with left ventricular hypertrophy and normal coronary arteries, with $\mathrm{T}$ wave inversion being asymmetrical in $\mathbf{9 5 \%}$ of these patients and that flat ST segment depression with or without $\mathrm{T}$ wave inversion, and isolated $T$ wave changes can be produced by left ventricular hypertrophy without coronary artery disease.

We thank Prof $S M$ Cobbe, Prof A R Lorimer, Prof J H McKillop, Dr F G Dunn, and Dr I Hutton for allowing their patients to be studied and/or for their co-operation in other patients to be studied
aspects of this work.

1 Carter WA, Estes EH. Electrocardiographic manifestations of ventricular hypertrophy: a computer study of ECGanatomic correlations in 319 cases. Am Heart J 1964, 68:173-82.

2 Devereux RB, Reichek N. Repolarisation abnormalities of left ventricular hypertrophy. Clinical, echocardiographic and haemodynamic correlates. $J$ Electrocardiol. 1982 15:47-54.

3 Kannel WB, Gordon T, Castelli WP, Margolis JR. Electrocardiographic left ventricular hypertrophy and risk of coronary artery disease. Ann Intern Med 1970;72:813-22.

4 Pringle SD, Macfarlane PW, McKillop JH, Lorimer AR, Dunn FG. Pathophysiologic assessment of left ventricular hypertrophy and strain in asymptomatic patients with hypertrophy and strain in asymptomatic patients with 1377-81.

5 Watts MP, Shoat DB. Trends in electrocardiograph design. J IERE 1987;57:140-50

6 Devereux RB, Reichek N. Echocardiographic determination of left ventricular mass in man. Circulation 1977;55:613-8.

7 DuBois D, DuBois EF. A formula to estimate approximate surface area if height and weight be known. Arch Intern Med 1916;17:863-71.

8 Devereux RB, Lutas EM, Casale PN. Standardisation of Mmode echocardiographic left ventricular anatomic measurements. J Am Coll Cardiol 1984;4:1222-30.

9 Milliken JA, Macfarlane PW, Lawrie TDV. Enlargement and hypertrophy. In: Macfarlane PW, Lawrie TDV, eds. Comprehensive electrocardiology. Oxford: Pergamon, 1989:631-70.

10 Rykert HE, Hepburn J. Electrocardiographic abnormalities characteristic of certain cases of arterial hypertension. $\mathrm{Am}$ characteristic of certain case

depression or isolated $T$ wave changes in patients with left ventricular hypertrophy and normal coronary arteries is probable latent myocardial ischaemia or a variant of left ventricular strain. However, we cannot rule out a structural abnormality in the myocardium, 